Gut, 1987, 28, 883-887

\title{
Twenty four hour oesophageal acidity in achalasia before and after pneumatic dilatation
}

\author{
H L SMART, P N FOSTER, D F EVANS, B SLEVIN, AND M ATKINSON \\ From the Department of Surgery, University Hospital, Queen's Medical Centre, Nottingham
}

SUMMARY Radiotelemetric ambulatory 24 hour oesophageal $\mathrm{pH}$ measurement was carried out in 17 patients with symptomatic manometrically proven achalasia before and after pneumatic dilatation. Before dilatation an abnormally high percentage acid exposure time was present but typical episodes of gastro-oesophageal reflux occurred in only one patient. In nine patients who had a oesophageal food residue these values were significantly greater than those found in the remaining patients without retained food. Analysis of oesophageal food residues revealed that the low $\mathrm{pH}$ was predominantly caused by lactic acid. These findings indicate that preoperative oesophageal $\mathrm{pH}$ studies do not offer a valid means of selection of patients in whom an antireflux procedure should be combined with cardiomyotomy. Repeat studies one week after pneumatic dilatation showed a fall in the acid exposure time in patients with initial food residue presumably resulting from improved oesophageal emptying after treatment. A significant rise in acid exposure time after dilatation was seen in those patients without initial food residue presumably resulting from gastro-oesophageal reflux after therapeutic disruption of the lower oesophageal sphincter. Troublesome symptoms of gastro-oesophageal reflux were, however, uncommon after bag dilatation.

Treatment of achalasia by forceful dilatation or surgical cardiomyotomy produces good relief of symptoms but may result in gastro-oesophageal reflux.' Few studies have assessed the oesophageal $\mathrm{pH}$ before treatment but it has been shown that gastro-oesophageal reflux may occur ${ }^{23}$ and this has led to the suggestion that in these patients an antireflux procedure should be done at the same time as cardiomyotomy. ${ }^{3}$ In order to investigate oesophageal $\mathrm{pH}$ further and to assess the effect of forceful dilatation we have carried out 24 hour oesophageal $\mathrm{pH}$ measurements in a group of patients before and after treatment of achalasia by pneumatic dilatation of the cardia.

\section{Methods}

PATIENTS

Seventeen patients ( 11 men) aged between 16 and 74

Address for correspondence: Professor M Atkinson, Department of Surgery, University Hospital. Queen's Medical Centre. Nottingham.

Received for publication 11 November 1986. years, with symptomatic achalasia proven by manometry and radiology were studied. Twelve patients were newly diagnosed as having achalasia and had received no previous treatment while the remaining five had previously undergone pneumatic dilatation of the cardia on one or more occasions but had developed recurrence of dysphagia.

All patients were admitted to hospital for their pretreatment assessment and this allowed the diagnosis of achalasia to be confirmed in new patients. After an overnight fast the patients underwent 24 hour oesophageal $\mathrm{pH}$ monitoring using a $\mathrm{pH}$ sensitive radio telemetric capsule and portable receiving equipment (MR 1000 solid state system, Oxford Medical Systems Ltd). ${ }^{+}$The capsule was placed $5 \mathrm{~cm}$ above the proximal end of the lower oesophageal sphincter as identified manometrically and tethered using a fine nylon thread taped to the cheek. Signals from the capsule were detected by an aerial belt around the chest connected to a solid state recorder. For the duration of the study patients were encouraged to be fully ambulant in the hospital 
environment and food with a $\mathrm{pH}$ value of less than 5 was avoided. At the end of the study the data was played back using a micro processor controlled playback unit linked to a standard digital printer. Data were analysed by calculating the time below $\mathrm{pH} 5$ and below $\mathrm{pH} 4$ expressing each as a percentage of the total, day and night recording period thus obtaining a percentage acid exposure time (\%AET).

After a 12 hour fast the patients underwent endoscopy and pneumatic dilatation of the cardia using the Rider-Moeller bag under general anaesthesia. ${ }^{5}$ The presence of food residue in the oesophagus was noted and a sample collected for measurement of its $\mathrm{pH}$, titratable acidity and organic acid content using gas liquid chromatography.

One week after pneumatic dilatation the 24 hour oesophageal $\mathrm{pH}$ study was repeated.

\section{STATISTICAL ANALYSIS}

The \%AET was calculated for patients before and after pneumatic dilatation. The values were compared between groups of patients using the Mann Whitney $U$ test for non-parametric data.

\section{Results}

For purposes of analysis and comparison the patients were divided into two groups; the nine with oesophageal food residue at endoscopy and the remaining eight without retained food. Before pneumatic dilatation patients with food residue had a greater $\%$ AET for both $\mathrm{pH}$ values than did patients without retained food (Fig. 1). Analysis of the oesophageal residue obtained from patients with retained food revealed this to be acidic (median $\mathrm{pH} 3 \cdot 8$, range $3 \cdot 5-4 \cdot 0)$. The acidity of the residue was found to be largely caused by the lactic acid content which accounted for between 91 and $97 \%$ of the total acid.

After pneumatic dilatation no food residues remained and acid exposure tended to be greater in patients with than in those without initial food residue but this difference was only significant for nocturnal $\mathrm{pH}$ measurement (Fig. 2). Those patients with initial food residue showed a fall in total \% AET for both $\mathrm{pH}$ values after pneumatic dilatation. These changes were less marked at night than during the day (Fig. 3). In those patients without initial food residue, pneumatic dilatation produced a significant increase in total and nocturnal \%AET for $\mathrm{pH}<5$, to reach pathological levels. There was, however, no change in the value for $\mathrm{pH}<4$ (Fig. 4).

Typical episodes of gastro-oesophageal reflux (defined as a sharp fall in $\mathrm{pH}$ of at least $2 \mathrm{pH}$ units to below $\mathrm{pH} 5$ or $\mathrm{pH} 4$ ) before treatment occurred in only one patient who had a relatively normal calibre oesophagus without retained food. Other recordings from patients without food residue were essentially

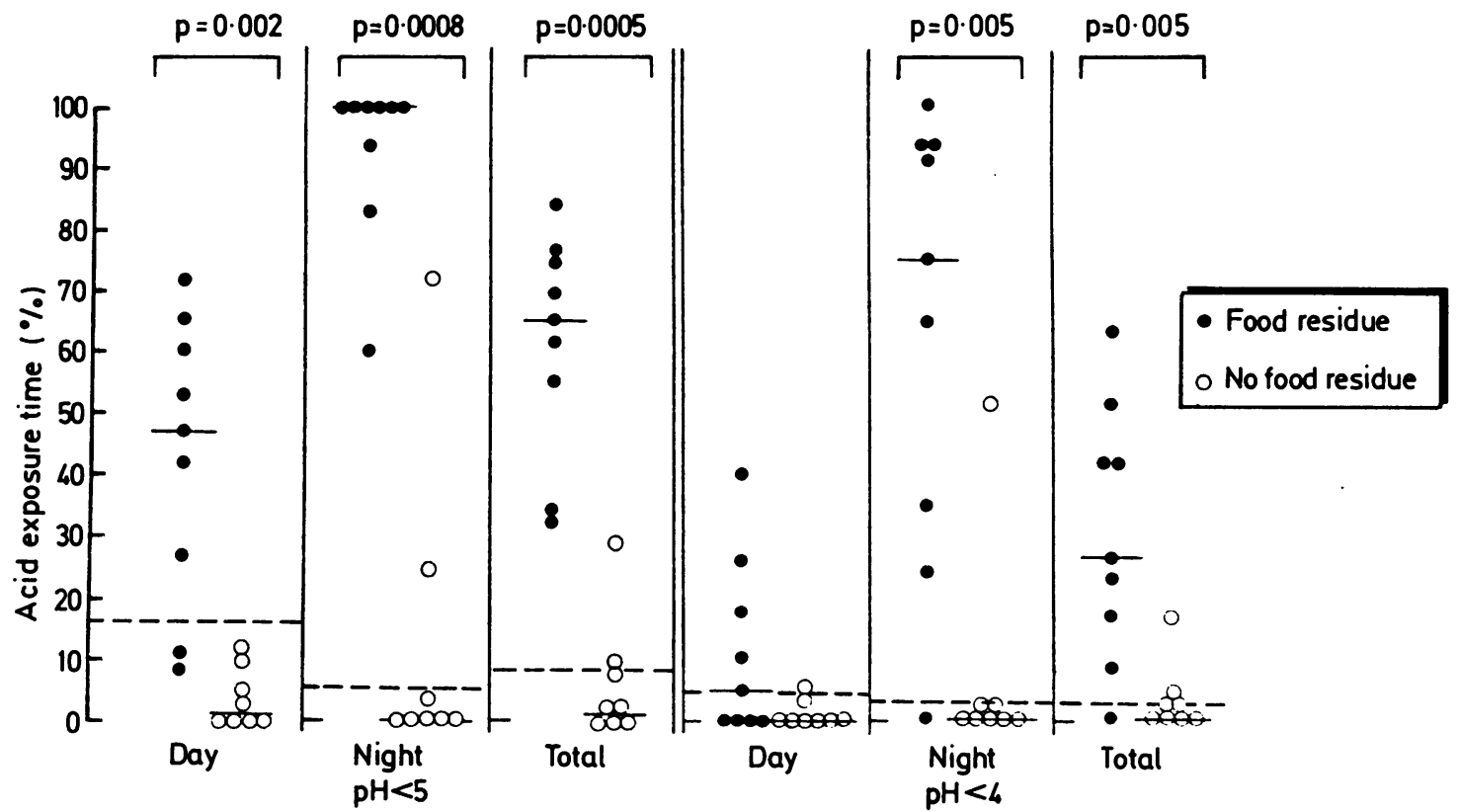

Fig. 1 Oesophageal acid exposure time below pH5 and below pH4 before dilatation in relation to residual food content in the oesophagus. Shaded circles represent values in patients with and open circles those without a food residue. Solid lines show the median values and attempted lines show the upper limits of the normal range. 


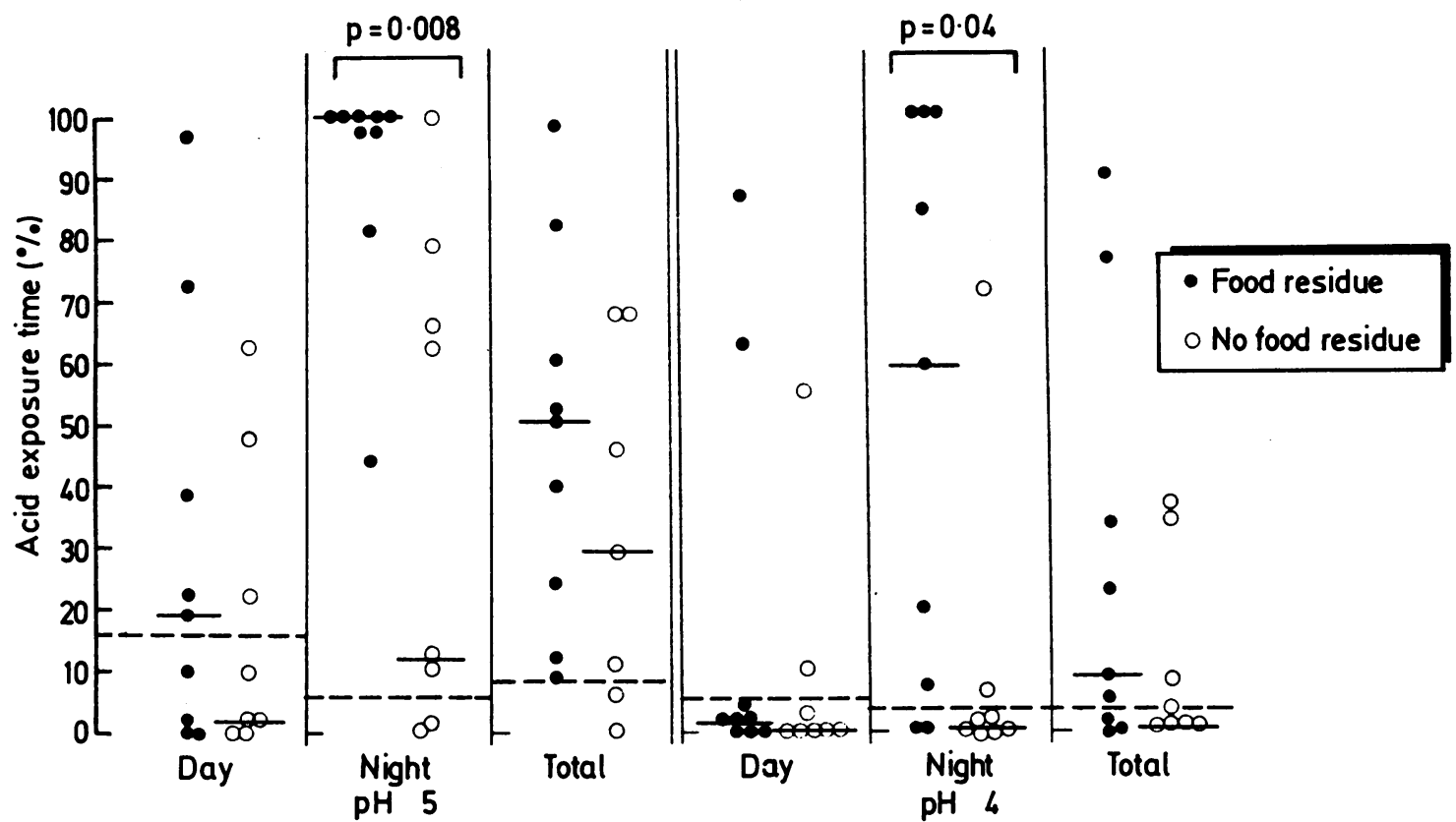

Fig. 2 Oesophageal acid exposure time below pH5 and below pH4 after pneumatic dilatation in relation to residual food content in the oesophagus before treatment. Shaded circles represent values in patients with and open circles those without a food residue. Solid lines show the median values and interrupted lines show the upper limit of the normal range.

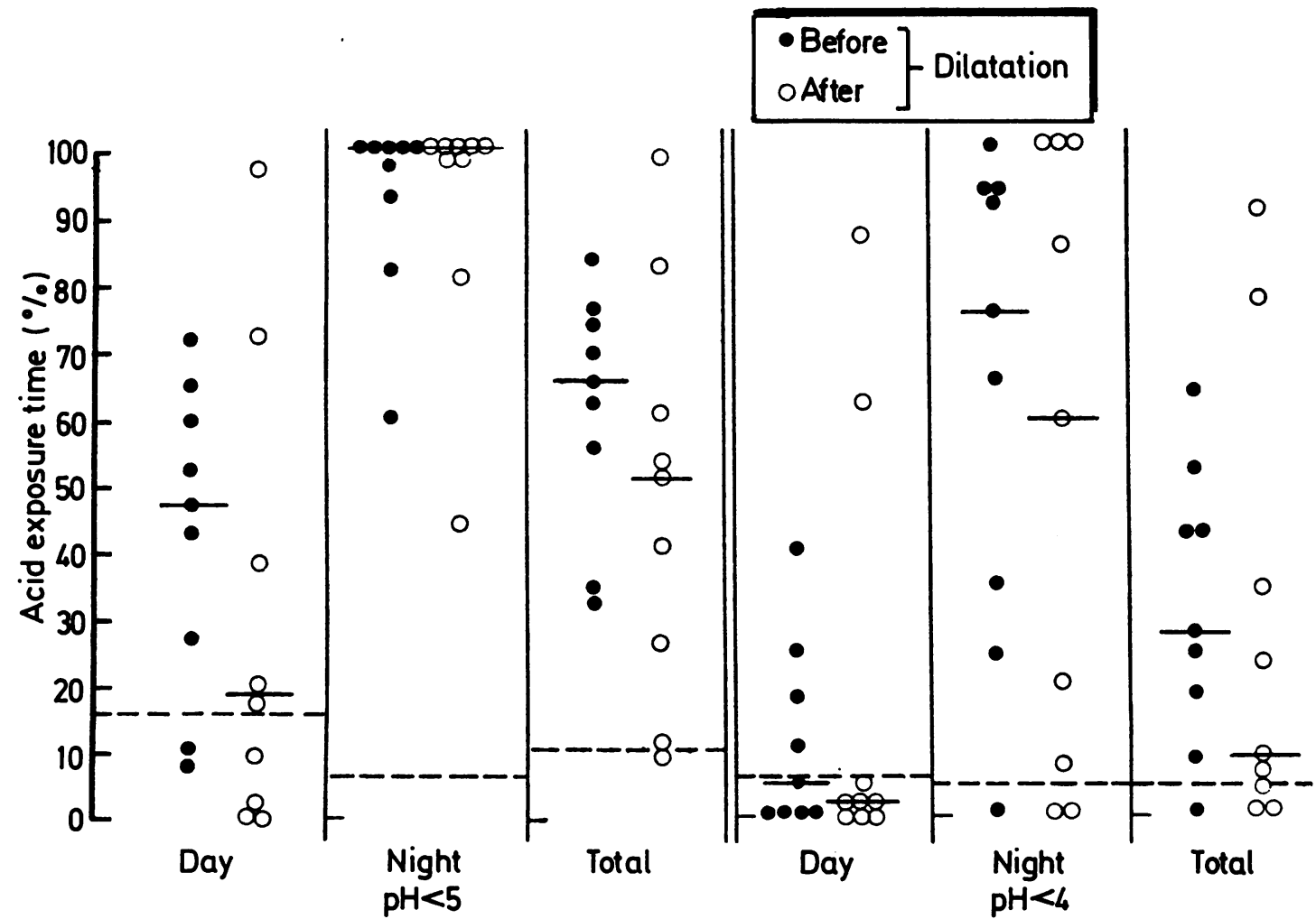

Fig. 3 Oesophageal acid exposure time below pH5 and below pH4 before (shaded circles) and after (open circles) pneumatic dilatation in patients with a pretreatment food residue in the oesophagus. Solid lines show the median values and interrupted lines show the upper limit of the normal range. 


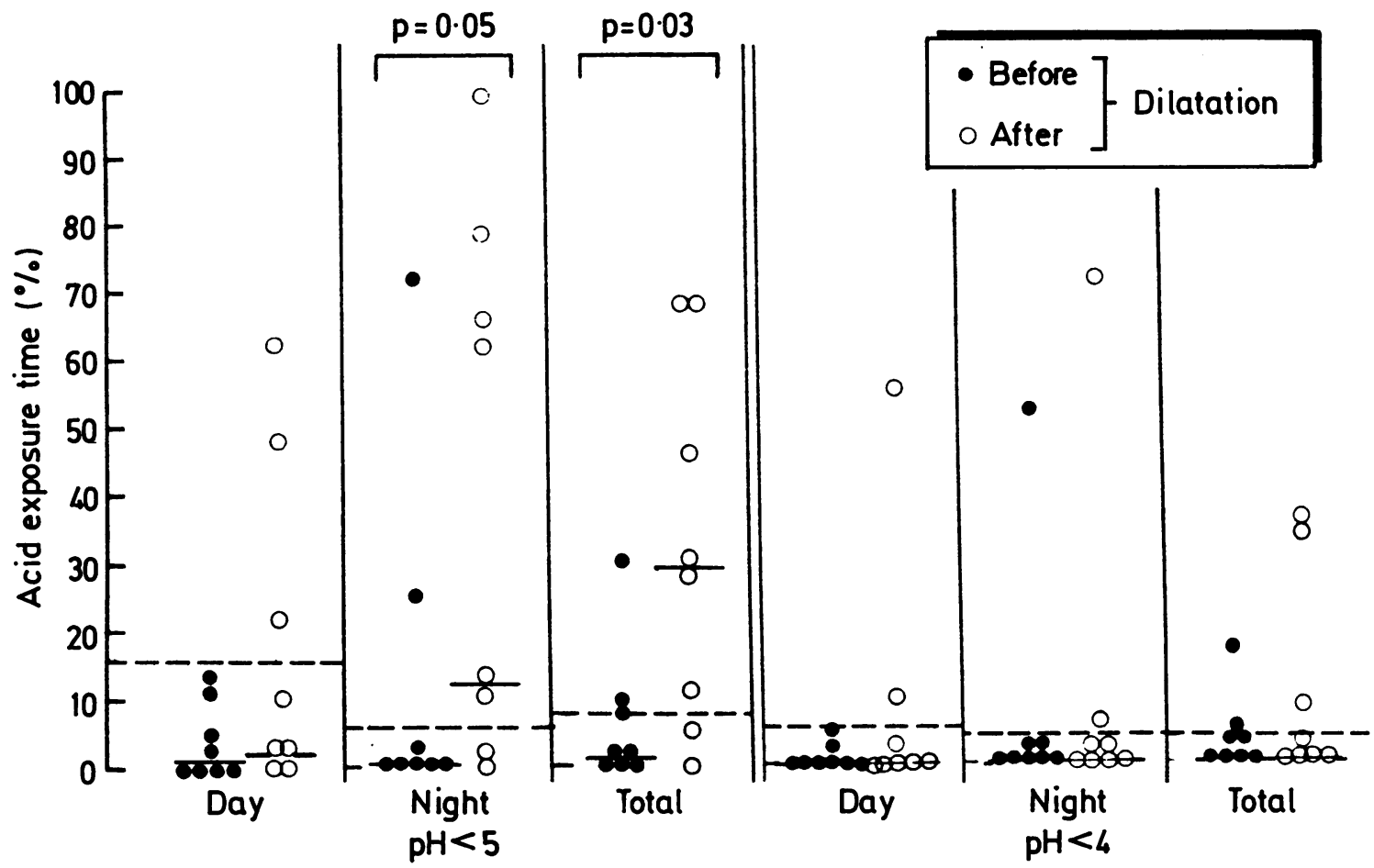

Fig. 4 Oesophageal acid exposure time below pH5 before (shaded circles) and after (open circles) pneumatic dilatation in patients without a pretreatment food residue in the oesophagus. Horizontal lines show the median values and interrupted lines show the upper limit of the normal range.

normal but in those with retained food a low basal oesophageal $\mathrm{pH}$ was common (values between $\mathrm{pH} 4$ and 5). After dilatation no increase in typical episodes of gastro-oesophageal reflux was observed but in some patients the oesophageal basal $\mathrm{pH}$ tended to be lower than in the pretreatment study (values between $\mathrm{pH} 3$ and 4 ).

\section{Discussion}

Gastro-oesophageal reflux may occasionally precede the development of achalasia' and has been reported to occur in between a quarter and a half of patients with established achalasia as revealed by oesophageal $\mathrm{pH}$ recording. ${ }^{23}$ In one of these studies, however, ${ }^{3}$ an associated hiatal hernia was often present which is normally a rare finding in achalasia ${ }^{7}$ and may have predisposed to reflux. In the other' a less physiological method than those now available was used. The preoperative finding of high oesophageal acidity has been used to select patients in whom cardio myotomy should be supplemented by an antireflux procedure. ${ }^{3}$

The present study indicates that gastrooesophageal reflux is distinctly uncommon in symptomatic achalasia before treatment and typical episodes of gastro-oesophageal reflux were recorded in only one of our 17 patients. Here the frequency duration index of gastro-oesophageal reflux ${ }^{x}$ was within the physiological as opposed to pathological episodes of reflux. Our findings suggest that a high pretreatment level of oesophageal acidity in achalasia is much more likely to be the result of lactic acid produced by fermentation of retained food residues; pretreatment oesophageal exposure was significantly higher in those patients with oesophageal food retention than in those without and the slow downward drift of oesophageal $\mathrm{pH}$ to reach a level corresponding with that caused by lactic acid in aspirated food residues together with the absence of episodic changes both support this view. Preoperative oesophageal $\mathrm{pH}$ monitoring is therefore an invalid method for the selection of patients requiring an antireflux procedure at the time of cardiomyotomy.

In the absence of coordinated oesophageal peristalsis in achalasia the effect of posture on oesophageal emptying is evident. In those patients with oesophageal food retention before treatment and in both groups after treatment, nocturnal oesophageal 
acid exposure was greater than that during the day. This reflects not only the importance of gravity but also the hydrostatic effect of food and fluids in enhancing oesophageal transit producing a comparable disorder to that seen in systemic sclerosis."

Our findings indicate that some degree of gastrooesophageal reflux is common after pneumatic dilatation for achalasia. Such reflux is, however, commonly asymptomatic. In only two of the 17 patients studied did symptoms occur and even these were transient and responsive to conservative treatment without recourse to long term $\mathrm{H} 2$ receptor antagonist drugs. Symptomatic reflux after bag dilatation appears to be much less common and disabling than after cardiomyotomy ${ }^{1}$ where peptic oesophageal stricture is a recognised complication.

\section{References}

1 Vantrappen G, Janssens J. To dilate or to operate? That is the question. Gut $1983 ; 24: 1013-9$.
2 Peyton M, Greenfield L, Elking R. Cardiomyotomy and hiatal herniorrhaphy. Am J Surg 1974; 128: 786-90.

3 Bjork S, Dernevik L, Gatzinsky P, Sandberg N. Cardiomyotomy and anti-reflux procedures. Acta Chir Scand 1982; 148: 525-9.

4 Evans DF, Jones JA, Hardcastle JA. Ambulatory monitoring of gastro oesophageal $\mathrm{pH}$ using a radiotelemetric capsule and a new solid state recorder with automated analysis. Gastroenterology 1985; 88: 1376.

5 Fellows IW, Ogilvie AL, Atkinson M. Pneumatic dilatation in achalasia. Gut 1983; 24: 1020-3.

6 Smart HL, Mayberry JF, Atkinson M. Achalasia following gastro-oesophageal reflux. $J R$ Soc Med 1986; 79: 71-3.

7 Binder HJ, Clemett AR, Thayer WR, Spiro HM. Rarity of hiatus hernia in achalasia. $N$ Engl J Med 1965; 272: 680-2.

8 Branicki FJ, Evans DF, Jones JA, Ogilvie AL, Atkinson M, Hardcastle JD. The evaluation of oesophageal reflux using a frequency duration index (F.D.I.) Br J Surg 1984; 71: 425-30.

9 Atkinson M, Sumerling MD. Oesophageal changes in systemic sclerosis. Gut 1966; 7: 402-8. 